THE ASTROPHYSICAL JOURNAL SUPPLEMENT SERIES, 90:753-764, 1994 February

(C) 1994. The American Astronomical Society. All rights reserved. Printed in U.S.A.

\title{
RADIO EVIDENCE FOR NONTHERMAL PARTICLE ACCELERATION ON STARS OF LATE SPECTRAL TYPE
}

\author{
KENNETH R. LANG \\ Department of Physics and Astronomy, Tufts University, Medford, MA 02155 \\ Received 1993 March 1; accepted 1993 May 19
}

\begin{abstract}
Radio evidence for nonthermal particle acceleration on stars of late spectral type ( $G, K$, and $M)$ is reviewed, including the pre-main-sequence T Tauri stars, the dwarf $M$ flare stars, and the RS CVn binary stars. Both the long-lasting radio emission and the transient radio flares can be attributed to nonthermal electrons accelerated in magnetically confined coronae, radiating by either incoherent gyrosynchrotron radiation or by coherent processes. Related observations of the Sun provide the framework for interpreting the radio emission of late-type stars. We review recent VLBI observations of single and binary stars, observations of rapid acceleration and dynamic spectra of dwarf $\mathrm{M}$ flare stars, and models for long-lasting radio emission from dwarf $\mathrm{M}$ flare stars and RS CVn stars. The VLBI data provide evidence for nonthermal particle acceleration in large-scale magnetospheres several times larger than the stellar radius for T Tauri and RS CVn stars. In contrast, rapid radio flares on dwarf $M$ stars require sources much smaller than the visible star in size, and this is consistent with the VLBI data.

Dynamic spectra suggest particle acceleration by relativistic electron beams on dwarf $M$ stars. The long-lived radio radiation of dwarf $M$ stars and $R S C V n$ stars requires more-or-less continuous particle acceleration over periods of hours, perhaps by persistent low-amplitude flaring.
\end{abstract}

Subject headings: acceleration of particles — binaries: close — radio continuum: stars — stars: flare stars: late-type - stars: pre-main-sequence

\section{INTRODUCTION}

\subsection{Magnetic Activity and Radio Diagnostics of Late-Type Stars}

Soft X-ray observations over the past decade have shown that virtually all cool stars of late spectral type ( $G, K$, and $M)$ are sources of strong thermal X-ray emission, attributed to a coronal plasma at temperatures between a million and ten million degrees. We know that the Sun has a million-degree corona that is constrained by magnetic fields, and trapped within coronal loops. So, we might expect that magnetic fields play an important role in confining and determining the structure of coronae of late-type stars, particularly when the hot plasma cannot be bound by the star's gravity (see Kundu et al. 1987 for flare stars); but the detailed geometry of the magnetically confined plasma is unknown.

In the solar case, the magnetic fields are assumed to be generated by internal dynamo action involving the interaction of rotation and convection. Stars later than spectral type $F$ have deep convection zones which, when combined with rapid rotation, are believed to generate strong magnetic fields via the dynamo effect. Greater magnetic activity may, in fact, play a role in the enhanced rate of coronal heating that gives rise to the luminous, thermal X-ray radiation from these stars.

Cool stars of late spectral type can also emit intense radio radiation that indicates particle acceleration to nonthermal energies. It is useful to classify this radio emission into transient flares with time scales of less than 1 hour, and long-lasting quiescent radiation that slowly varies and lasts more than 1 hour. The observed radio emission on both short and long time scales is at least 1000 times less energetic than that in the $\mathrm{X}$-ray region of the electromagnetic spectrum. However, the radio data provide an important diagnostic tool for magnetic fields and accelerated particles.

Observations of the radio brightness temperature, polarization, and temporal and spectral characteristics provide important constraints on the radiation and acceleration mechanisms. These data can specify the coronal magnetic field strength, the thermodynamic state of the coronal plasma, and the properties of the high-energy, nonthermal radio-emitting electrons. In fact, radio astronomy remains the only technique by which the coronal magnetic field can be measured directly, thereby providing unique insight to the magnetic energy that is probably required to briefly power explosive stellar flares, to continuously produce long-lasting nonthermal radiation from stars, and to accelerate the high-speed electrons that are required for nonthermal processes.

In general, both the quiescent and flaring radio radiation of late-type $(G, K$, or $M)$ stars can be attributed to nonthermal electrons in magnetically confined coronae by either coherent or incoherent processes. By way of comparison, there are apparently no detected nonthermal radio stars for stellar spectral types between those of the magnetized B stars and spectral type G0; stars in this radio gap do not efficiently convert their internal energy into magnetic activity and particle acceleration. (The winds of giant stars of early spectral type do emit thermal radio emission, but this review concerns nonthermal radio radiation of stars of late spectral type.)

\subsection{Correlations}

The absolute luminosity of the thermal X-ray radiation of late-type stars $(G$ to $M)$ increases with the square of the equatorial rotational velocity of the star, being greatest for the binary 
RS CV $n$ stars that are tidally locked into rapid rotation (Pallavicini et al. 1981); but the dispersion within individual classes of objects, such as RS CVn stars or dwarf $M$ flare stars, does not permit a well-defined correlation with rotation rate within that class (also see Pallavicini, Stella, \& Tagliaferri 1990). The greater speed of rotation presumably results in enhanced magnetism by internal dynamo action, resulting in greater coronal heating, X-ray emission and radio radiation. Slee \& Stewart (1989) additionally showed that radio stars with active chromospheres, including the RS CVn stars, have a radio surface brightness that is correlated with rotation rate. Drake, Simon, \& Linsky $(1989,1992)$ found a significant correlation of both the $\mathrm{X}$-ray and radio luminosity with the rotation rate of $\mathrm{RS}$ CVn stars, and they found strong positive correlations of the quiescent radio luminosity and X-ray luminosity of these stars.

Güdel (1992) showed a similar close correlation of X-ray and radio luminosities for magnetically active, main-sequence $\mathrm{K}$ stars. Such a linear correlation has also been found for dwarf M stars (Güdel et al. 1993). Most recently, Güdel \& Benz (1993) have demonstrated that the positive correlation between the long-lasting $X$-ray and radio luminosities (close to proportionality) exists for a variety of late-type, main-sequence stars independent of age, spectral class, binarity, rotation, or photospheric/chromospheric activity. These correlations suggest that the coronal heating of thermal X-rayemitting electrons is closely linked to the acceleration of the nonthermal, energetic electrons that produce the radio emission. Such an association between thermal X-ray radiation and nonthermal radio emission is probably related to magnetic activity on these stars, and perhaps related to internal rotation and convection.

\subsection{T Tauri Stars, Dwarf M Flare Stars, and RS CVn Stars}

In this paper, I review radio evidence for nonthermal particle acceleration on stars of late spectral type $(G, K$, and $M)$. They include the pre-main-sequence $T$ Tauri stars, the young dwarf $M$ flare stars and the RS Canum Venaticorum, or RS $\mathrm{CVn}$, stars, whose bulk properties are compared with those of the Sun in Table 1. We note at the outset that the Sun is a somewhat unusual star, with a relatively slow rotation and low $\mathrm{X}$-ray luminosity, and that its radio emission is also relatively weak.

The T Tauri stars are typically between 10,000 and a million years old, and of late spectral type, usually $K$; they are often found embedded within dark clouds of gas and dust such as those found in Ophiuchus and Taurus-Auriga. They were initially identified by intense emission lines of hydrogen; but more recently weak-line T Tauri (WTT) stars have been found in the same star-forming regions, often because of their intense X-ray radiation. Radio radiation from WTT stars is relatively intense when compared with the Sun, and can vary by at least an order of magnitude on time scales of days to years. The varying radio radiation from one similar rapidly rotating star was most intense during passage of a large star spot, suggesting that electrons are accelerated nearly continuously in regions of strong coronal magnetic fields above star spots (Lim et al. 1992). Nonthermal radio radiation has also been detected from the classical pre-main-sequence binary T Tauri (Phillips et al. 1993).
Although all young main-sequence stars probably begin their life flaring, and continue flaring throughout most of their life, the term flare star designates a particular group of dwarf, or main-sequence, red stars, generally of spectral type $\mathbf{M}$, with extraordinarily bright, transient (seconds to minutes) optical brightenings. They are 0.1 to 1 billion years old.

The RS CVn stars are late-type binary stars whose enhanced coronal activity results in exceptionally intense X-ray and radio emission. This activity is thought to be closely connected with strong magnetic fields on stars that are tidally locked into rapid synchronous rotation. These stars usually have a mainsequence dwarf, or subgiant, primary of spectral type $F$ or $G$ and a somewhat cooler, usually more massive and evolved, secondary star that is typically an early $\mathrm{K}$ subgiant.

For background, a general review of flares on the Sun and other stars is given by Haisch, Strong, \& Rodono (1991), while Hjellming (1988) has focused on radio-emitting stars including topics that are not discussed here (such as stellar winds, catacylsmic variables and X-ray binaries). Radio and X-ray observations of pre-main-sequence stars are discussed by Feigelson, Giampapa, \& Vrba (1991). Lang (1990a) has reviewed recent radio observations of the Sun; radio emission from dwarf M flare stars has been discussed by Bastian (1990) and by Lang (1990b), and Kuijpers (1989) reviewed emission mechanisms for stellar radio flares.

Observing coordinates and physical data for specific stars can be found in Lang (1991). Radio surveys of either starforming regions and/or specific T Tauri stars have been completed by André, Montmerle, \& Feigelson (1987), André et al. (1992), Phillips et al. (1991), Stine et al. (1988), and White, Pallavicini, \& Kundu (1992).

\section{THERMAL HEATING AND NONTHERMAL PARTICLE ACCELERATION ON THE SUN}

The Sun is the only star close enough to permit the detailed radio-wavelength scrutiny needed to localize the regions of thermal heating and nonthermal particle acceleration. Observations of radio emission from the Sun have also led to the discovery of a kaleidoscopic variety of phenomena, including positive and negative frequency drifts, sudden reductions, quasi-periodic pulsations, microwave spike bursts and longlasting nonthermal emission (McLean \& Labrum 1985). Each of these phenomena have observational counterparts on latetype stars. Moreover, the theoretical framework for interpreting the radio emission from late-type stars has its foundations in the theory of solar radio radiation, including gyroresonant absorption, and the gyrosynchrotron, synchrotron, and coherent emission processes. We therefore first provide a brief overview of the Sun's radio radiation, which establishes a context for interpreting thermal heating and nonthermal particle acceleration on late-type stars.

\subsection{Long-Lasting Thermal Solar Radio Radiation}

The hot, million-degree corona of the Sun radiates thermal bremsstrahlung detectable at both soft X-ray and radio wavelengths; coronal magnetic fields shape, constrain and mold the high-temperature material, giving rise to the ubiquitous coronal loops in which it is concentrated. The development of aperture synthesis telescopes like the Very Large Array (VLA) has 
TABLE 1

LATE-TyPe StaRs EMITting Nonthermal Radio Radiation

\begin{tabular}{|c|c|c|c|c|}
\hline Parameter & $\begin{array}{c}\text { Sun } \\
\text { (Main-Sequence) }\end{array}$ & $\begin{array}{l}\text { Weak-Lined T Tauri } \\
\quad \text { (WTT) Stars } \\
\text { (Pre-Main-Sequence) }^{\mathbf{a}, \mathbf{b}}\end{array}$ & $\begin{array}{c}\text { Flare Stars } \\
\text { (Main-Sequence) }^{\mathrm{b}}\end{array}$ & $\begin{array}{l}\text { RS Canum Venaticorum } \\
\text { (RS CVn) Stars } \\
\text { (Tidally Locked, Evolved } \\
\text { Binary Stars) }\end{array}$ \\
\hline Age $(\mathrm{yr}) \ldots \ldots \ldots \ldots$. & $4.6 \times 10^{9}$ & $10^{5}-10^{6}$ & $10^{8}-10^{9}$ & $\approx 10^{10}$ \\
\hline Spectral class . ............. & $\mathrm{G} 2 \mathrm{~V}$ & $\mathrm{G}-\mathrm{K}$ & $\mathrm{dMe}$ & $\begin{array}{l}F \text { or } G \text { main-sequence dwarf } \\
\text { and early } K \text { subgiant }\end{array}$ \\
\hline Mass $M\left(M_{\odot}\right) \ldots \ldots$ & $1^{\mathrm{c}}$ & 1 & $0.06-0.80$ & $0.5-3$ \\
\hline Rotation velocity $V_{\text {rot }}\left(\mathrm{km} \mathrm{s}^{-1}\right) \ldots$ & 2.0 & 25 & $10-20$ & $20-100$ \\
\hline X-ray luminosity $L_{\mathrm{X}}\left(\mathrm{ergs} \mathrm{s}^{-1}\right) \ldots$ & $10^{27}-10^{28}$ & $10^{29}-10^{31}$ & $10^{27}-10^{29}$ & $10^{30}-10^{32}$ \\
\hline Flare luminosity $L_{f}\left(\mathrm{ergs} \mathrm{s}^{-1}\right) \ldots \ldots$ & $10^{29}-10^{30}$ & $10^{31}-10^{32}(\mathrm{X}$-rays $)$ & $10^{25}-10^{29}$ (optical) & f \\
\hline Flare energey $E_{f}$ (ergs) $\ldots \ldots \ldots \ldots$ & $10^{31}-10^{32}$ & $10^{35}-10^{36}$ (X-rays for hours) & $10^{27}-10^{35}$ & \\
\hline Examples .................... & & V410 Tau, V826 Tau & UV Ceti, AD Leo, YZ CMI & UX Arietis, HR 1099 \\
\hline
\end{tabular}

${ }^{a}$ Classical T Tauri (CTT) Stars with strong H-alpha emission and an infrared excess, indicating, respectively, a dense circumstellar envelope and a dust disk can also emit nonthermal radio radiation; the star $\mathrm{T}$ Tauri is an example.

b Celestial coordinates and physical parameters can be found in Lang 1991.

c $1 M_{\odot}=1.989 \times 10^{33} \mathrm{~g}$.

${ }^{\mathrm{d}} 1 L_{\odot}=3.8 \times 10^{33} \mathrm{ergs} \mathrm{s}^{-1}$.

e $1 R_{\odot}=6.96 \times 10^{10} \mathrm{~cm}$.

${ }^{\mathrm{f}}$ Radio flares 10 to 1000 times more intense than those of flare stars.

permitted ground-based observations of coronal loops, indicating that the thermal bremsstrahlung of the X-ray emitting plasma can also be detected at radio wavelengths (Lang 1990a; Lang et al. 1987a, b, 1993).

In the strong magnetic fields above sunspots, gyroresonance absorption by thermal electrons in the corona provides an important source of opacity, accounting for intense radio radiation at centimeter wavelengths in regions where $\mathrm{X}$-ray radiation can be at undetectable levels. Observations of polarized horseshoe or ring structures at $6 \mathrm{~cm}$ wavelength (Alissandrakis \& Kundu 1982; Lang \& Willson 1982) confirmed theoretical predictions of thermal gyroresonance emission above individual sunspots. Multiple-wavelength radio observations with high angular and spectral resolution are now used to delineate the distribution of this radiation at different heights along the legs of coronal loops and to specify the strength and structure of the magnetic field in the low corona (see Bogod et al. 1992 for an example). Systematic radio spectral observations of gyroresonant emission indicate that the magnetic field strength of the thermal plasma at the million-degree level above large sunspots is 75 to 80 percent of the magnetic field strength in the underlying photospheric sunspots (Lang et al. 1993).

Although the long-lived solar radio radiation is usually interpreted in terms of thermal processes, either as bremsstrahlung or as gyroresonance radiation at harmonics of the gyrofrequency, long-lasting nonthermal radio sources are also found on the Sun ( see $\S 2.5$ ); but they do not dominate solar radio emission. In this respect, the Sun differs from the late-type stars reviewed here, for their steady radio radiation requires nonthermal particle acceleration to boost it to detectable levels at their greater distances.

No one really understands what mechanisms heat the Sun's corona to a million degrees, thereby sustaining its hot thermal plasma. But current speculations about coronal heating may be related to particle acceleration on late-type stars. Parker
(1988) and Sturrock et al. ( 1990) have, for example, proposed solar coronal heating by low-level microflares in magnetic dipoles or small flaring loops that presumably mimic more intense flares in larger scale coronal loops found in active regions.

\subsection{Nonthermal Solar Radio Bursts}

On the Sun, a typical radio flare, or burst, at centimeter wavelengths includes the precursor, impulsive and decay phases, the total radio emission lasting only a few minutes or tens of minutes (Fig. 1). The flare can be triggered during the precursor stage by interacting and reconnecting coronal loops (Kundu et al. 1982; Kundu \& Lang 1985; Lang \& Willson 1986a), resulting in the rapid impulsive release of stored magnetic energy. During the impulsive phase, electrons are accelerated to energies from $100 \mathrm{keV}$ to $1 \mathrm{MeV}$, emitting gyrosynchrotron radiation that is detected at radio wavelengths. These nonthermal particles move through a coronal magnetic field of a few hundred gauss and emit at frequencies that are high harmonics of the gyrofrequency; hard X-rays are produced by similar nonthermal electrons when they hit the underlying chromosphere. This is followed by the more gradual release of energy when the coronal loops relax into a more stable configuration during the decay phase, accompanied by increased soft X-ray radiation.

The Sun is the only star close enough for a close-up radio inspection of the coronal magnetic structure during the various burst phases, indicating that the impulsive energy release and particle acceleration often take place at the apex of coronal loops (Fig. 2; Lang \& Willson 1986a), and that the precursor, impulsive and decay phases can occur in nearby but spatially separate coronal magnetic structures (Willson, Lang, \& Liggett 1990).

Solar radio bursts observed at meter wavelengths indicate that intense flares can provide a long-lived source of nonther- 


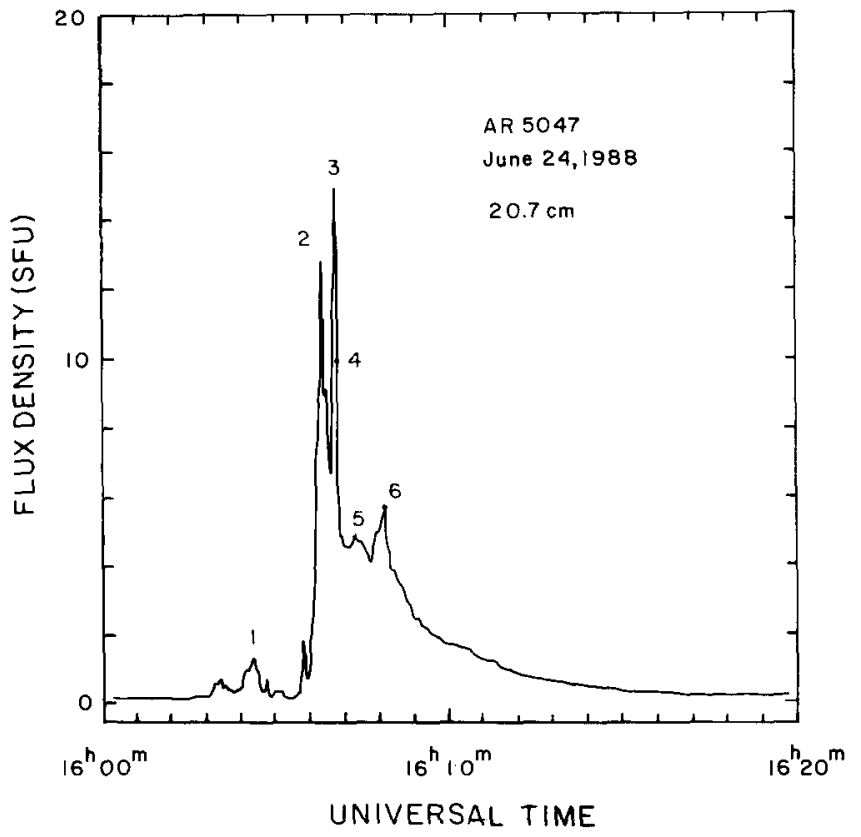

FIG. 1.-Time profile of a typical solar radio burst, or flare, consisting of a precursor (1) that occurs a few minutes before the onset of the impulsive burst emission $(2,3)$ that has rise times of less than 10 seconds, and the more gradual decay, or post-impulsive, phase $(4,5,6)$ lasting several minutes. VLA snapshot maps have shown that the precursor, impulsive and decay phases of this event occur in nearby, but spatially separate, coronal sources. (Adapted from Willson, Lang, \& Liggett 1990.)

mal electrons, sometimes lasting for hours and even days, giving rise to various forms of coherent nonthermal processes that can include highly circularly polarized radiation and brightness temperatures of $10^{9}$ to $10^{12} \mathrm{~K}$ (Mc Lean \& Labrum 1985).

\subsection{Temporal Fine Structure of Solar Radio Bursts}

Spike bursts are fairly common in solar flares observed at short centimeter wavelengths, or microwaves. The spikes have rapid rise times of a few milliseconds or less, and similarly brief durations. The quick release of radiation implies source sizes (from light travel time arguments) smaller than 1000 kilometers and brightness temperatures well in excess of $10^{10}$ degrees, indicating the presence of a coherent emission process. One such process, used to explain microwave spike bursts in solar flares, is the electron-cyclotron maser (Melrose \& Dulk 1982); maser is the acronym for microwave amplification by stimulated emission of radiation. According to the electron-cyclotron maser hypothesis, electrons are accelerated in the initial flare release near the loop apex, streaming down toward the footpoints of the magnetic loop. Some of these electrons mirror in the coronal loop; they can become unstable to the growth of electromagnetic waves at the gyrofrequency (and possibly harmonics thereof), tapping the available free energy.

Another type of temporal fine structure observed during solar radio bursts is quasi-periodic variations, or pulsations, that have been interpreted in terms of oscillations within magnetic flux tubes (Roberts, Edwin, \& Benz 1984). Small amplitude radial oscillations can be excited with periods of the order of the tube radius divided by the Alfven velocity. Observations of similar quasi-periodic variations in stellar radio emission can provide insights to the size, magnetic field and density of coronal loops on these stars.

Certain solar radio bursts also exhibit sudden reductions that have also been observed in the radio emission of dwarf $M$ flare stars ( see $\S 4.2$ ). They may also be related to radiation driven by a loss-cone instability (Benz \& Kuijpers 1976).

\subsection{Dynamic Spectra of Solar Radio Bursts}

Observations of solar radio burst intensity as a function of both frequency and time (dynamic spectra) provide direct information about particle acceleration processes on the Sun. Radio bursts designated as type II, for example, drift from high to low frequencies at rates of $\approx 1 \mathrm{MHz}$ per second, while those of type III have a drift rate of 10 to $100 \mathrm{MHz}$ per second (Mc Lean \& Labrum 1985). The type II bursts have brightness temperatures of $10^{7}-10^{11} \mathrm{~K}$, and are thought to be accelerated by shock fronts moving at 500 to 1000 kilometers per second. Type III bursts are generally brighter $\left(10^{8}-10^{15} \mathrm{~K}\right)$, and are attributed to rapid electron beams moving between 0.2 and 0.6 times the velocity of light.

Both positive and negative frequency drifts have been observed during solar bursts, respectively indicating disturbances propagating inward from the Sun and outward toward its visible surface. When both types of drift are observed, the wavelength which separates them marks the location of the particle acceleration process.

\subsection{Long-Lasting Nonthermal Solar Radio Radiation}

Comparisons of high-resolution radio images (VLA) and spectra (RATAN 600) indicate that nonthermal radio energy release mechanisms are also possible in quiescent, or nonflaring, solar active regions. That is, long-lived radio sources with high brightness temperatures $\geq 10^{7} \mathrm{~K}$ and steep radiation spectra are occasionally found that cannot be attributed to thermal bremsstrahlung or thermal gyroradiation (Fig. 3; Akhemedov et al. 1986). These sources occur above the magnetic neutral line in the underlying photosphere, and are associated with a multipolar (delta) configuration of the photospheric sunspots (Lang et al. 1993). They require nearly continual acceleration of energetic electrons by a yet unknown process. Although long-lived thermal radio radiation is more common on the Sun where bipolar magnetic structures predominate, longlived nonthermal radiation is much more prevalent on latetype stars ( see $\$ 5$ ).

\section{VLBI EVIDENCE FOR NONTHERMAL PARTICLE ACCELERATION ON LATE-TYPE STARS}

\subsection{VLBI of Single Stars}

Very Long Baseline Interferometry (VLBI) has been used to detect nonthermal radio radiation from more than a dozen single young, late-type ( $\mathrm{G}$ or $\mathrm{K}$ ) pre-main-sequence stars (see Phillips 1992 for a review). They usually show combinations of unresolved (less than 2 stellar radii) and resolved ( 5 to 20 stellar radii) radio-emitting regions (Phillips et al. 1991). The brightness temperatures range from $10^{7}$ to more than $10^{9} \mathrm{de}$ - 

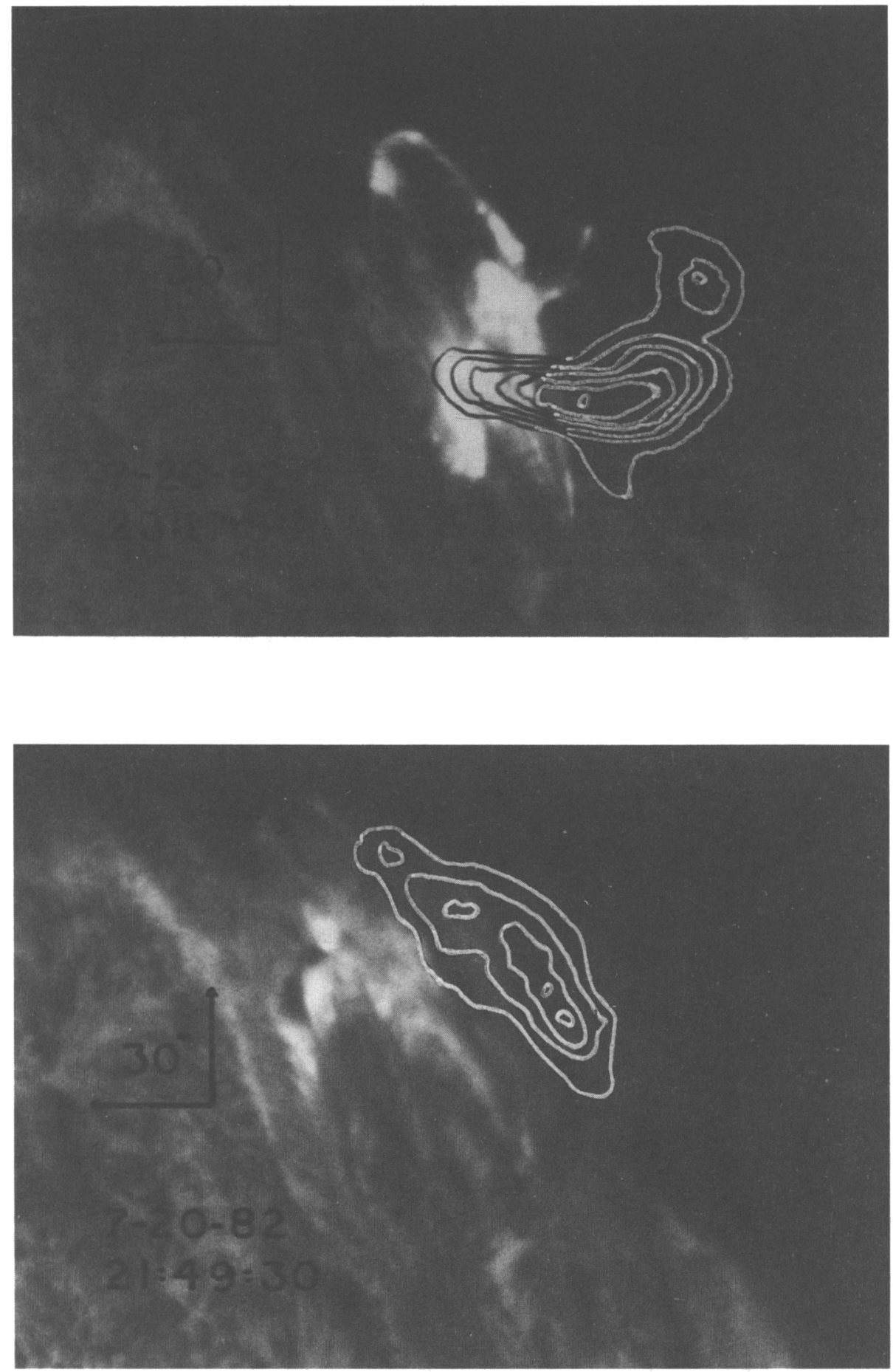

FIG. 2.- 10 second VLA snapshot maps of the impulsive phase of two solar bursts at $20 \mathrm{~cm}$ wavelength superposed on $\mathrm{H}$-alpha photographs of the optical flares taken at the same time at the Big Bear Solar Observatory. The $20 \mathrm{~cm}$ bursts originate near the tops of coronal loops that are about 40,000 kilometers above the flaring radiation seen at optical wavelengths. The western solar limb is visible in both photographs. (Adapted from Lang \& Willson 1986a.)

grees, suggesting that the radio radiation is nonthermal in origin (Phillips et al. 1991; Andre et al. 1991, 1992-thermal stellar winds would give much lower brightness temperatures.)

Examples of resolved stars include the young $\mathrm{K}$ star HD 283447 that apparently sustains nonthermal processes to 10 or 20 stellar radii, and the pre-main-sequence G-K star DoAr 21 whose radio emission may originate in magnetic structures of up to 25 stellar radii in diameter. The detection of low-level ( $2 \%$ to $4 \%$ ) circular polarization in the radio emission of these two weak-line $T$ Tauri stars is direct confirmation of the presence of magnetic fields in their coronae, and suggests optically thick gyrosynchrotron radiation of nonthermal electrons (White, Pallavicini, \& Kundu 1992).

The nonthermal radio radiation of the resolved structures of 

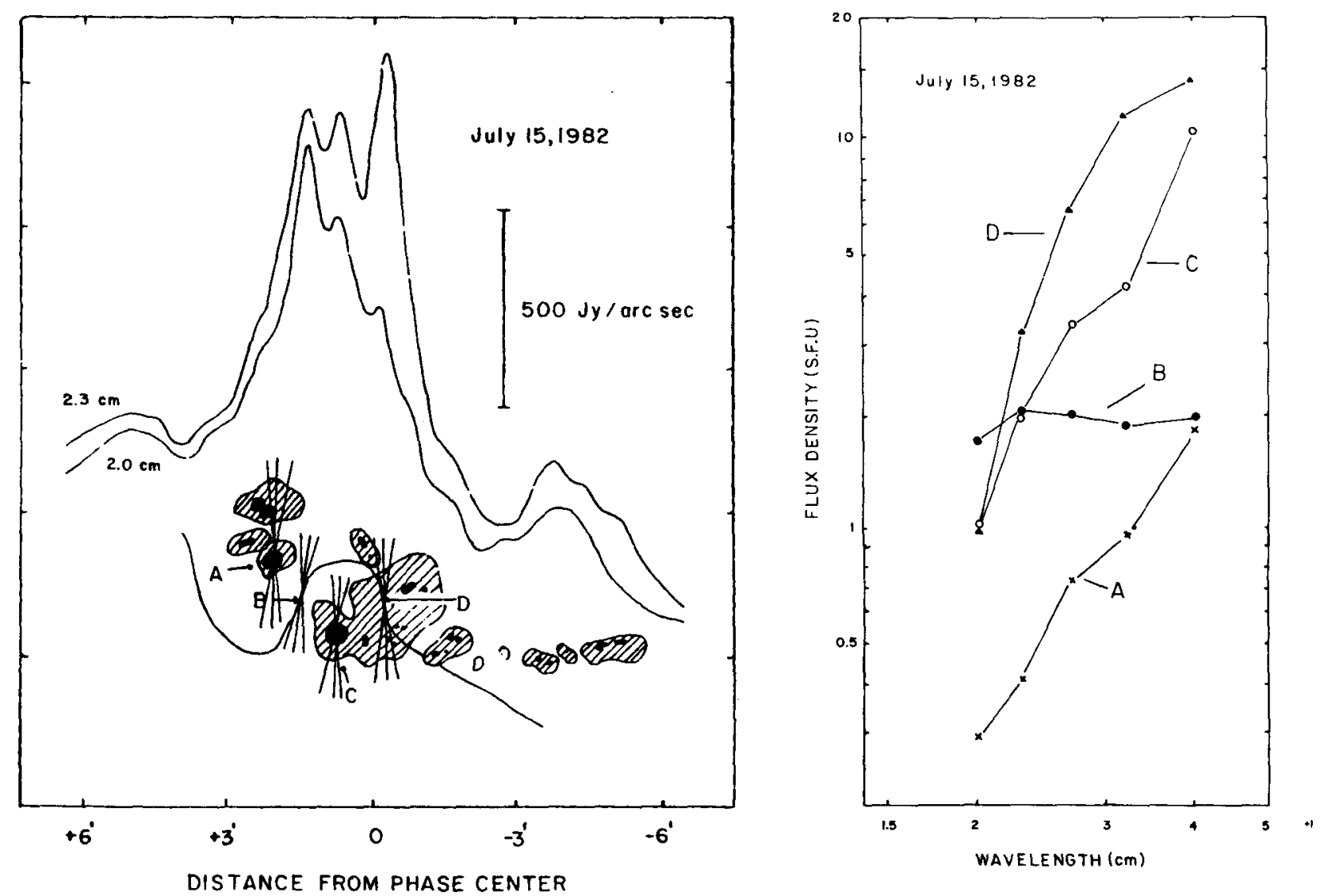

FIG. 3.-One-dimensional fan beam scans (left) of an active region, taken at different times with the RATAN 600, locate sunspot-associated sources A and $C$ above the sunspot umbrae (black); two other radio sources $B$ and $D$ are associated with the neutral line between sunspots of opposite magnetic polarity. The radio spectra ( right) of radio sources A and C are consistent with thermal gyroresonance absorption above sunspots, and the spectra of source B is consistent with optically thick thermal bremsstrahlung from coronal loops connecting sunspots of opposite magnetic polarity. A peculiar, long-lived nonthermal source (D) has a high brightness temperature ( 10 million degrees) and steep radiation spectrum that are difficult to explain by thermal processes. (Adapted from Akhmedov et al. 1986.)

late-type, pre-main-sequence stars apparently originates in large-scale magnetospheres several times larger than the stellar radius, probably by high-velocity electrons that emit gyrosynchrotron radiation; but the detailed mechanism for accelerating these nonthermal particles is not understood.

We do not have a clear VLBI picture of radio flares on premain-sequence stars, and there is an ongoing controversy over whether the relevant magnetic structures are significantly larger or smaller than a stellar radius.

The rise and decay time for centimeter emission from dwarf M flare stars can be shorter than the usual accumulation periods for the collection and fringe-fitting of VLBI systems, so the data must be carefully interpreted. Benz \& Alef (1991) have nevertheless used VLBI to show that the nonflaring radio radiation from the dwarf $\mathrm{M}$ star $\mathrm{YZCMi}$ is unresolved with an angular size less than 1.0 milliarcseconds, corresponding to a diameter of less than 1.7 times that of the visible star. The brightness temperature of more than $10^{9} \mathrm{~K}$ indicates nonthermal radiation, perhaps through the incoherent gyrosynchrotron mechanism or a coherent process. Alef et al. (1993) have also done VLBI of a stellar flare from the dwarf $M$ star EQ $\mathrm{Peg} / \mathrm{B}$, showing that this source is also largely unresolved, with size and brightness temperature limits comparable to those of
YZ CMi. Such compact radio sources with high brightness temperatures are also inferred from the rapid radio flares of dwarf M stars ( see $\S 4.1$ ).

\subsection{VLBI of Active Binary Stars}

Very Long Baseline Interferometery (VLBI) of stellar sources was initiated in the 1970s by observations of Algol, a semi-detached close binary (belonging to a multiple system) using the narrow-band Mark I data acquisition system (Clark et al., 1976); and developed in the 1980's with VLBI of RS $\mathrm{CVn}$ binary stars using the enhanced sensitivity of the wideband Mark III system and large-aperture antennas such as those of the Deep Space Network (Lestrade et al., 1988). The two types of active binary stars resolved with VLBI are illustrated in Figure 4.

The VLBI observations of RS CVn stars were motivated by both astrometric and astrophysical goals. In astrometry, precise radio positions, proper motions and parallaxes are obtained for the optically bright RS CVn systems, providing a link between the radio and optical celestial reference frames. In astrophysics, VLBI can provide direct measurements of the size, structure, and brightness temperature of radio-emitting 


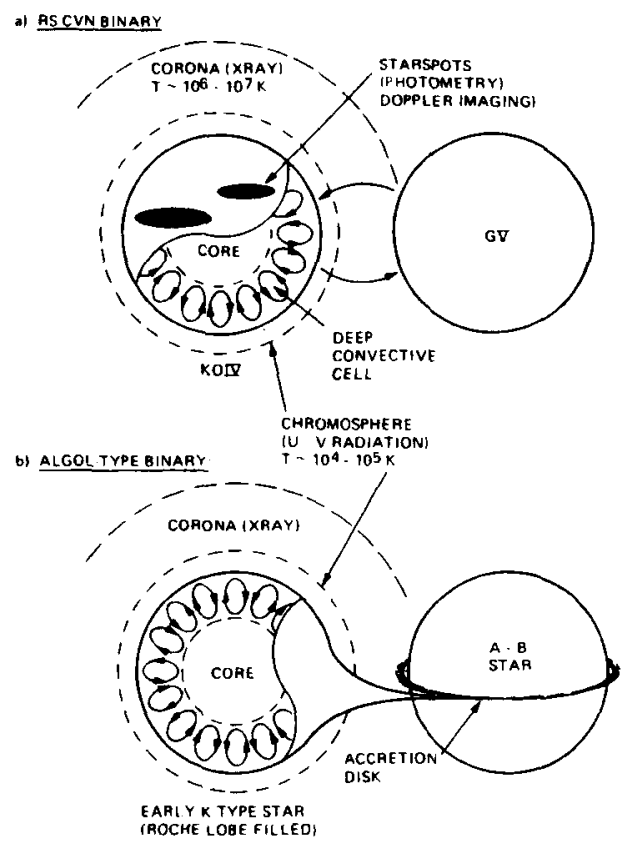

FIG. 4.-Active binary stars whose radio emission has been resolved with VLBI, indicating the presence of a halo component whose angular size of a few milli-arcseconds is comparable to the separation of the two stars. The RS CVn stars have big starspots suggesting large coronal loops, while one component of Algol overflows its Roche lobe leading to accretion of matter by a companion star. (Adapted from Lestrade 1988.) regions with angular sizes of a few milliarcseconds, or provide upper limits to this size with lower limits to the brightness temperature. A representative, current description of these astrophysical results can be found in Lestrade (1988).

The main VLBI result is that the radio emission from binary RS CVn stars exhibits a variable core-halo structure. The core is smaller than the $\mathrm{K}$-subgiant which is presumably the active star, while the halo component is comparable to the overall size of the binary system. The brightness temperature of the halo is typically $10^{8} \mathrm{~K}$, and that of the core exceeds $10^{10} \mathrm{~K}$. These high brightness temperatures suggest nonthermal mechanisms for both the halo and core components. Models for the long-lasting radio radiation from $\mathrm{RS} \mathrm{CVn} \mathrm{stars} \mathrm{are} \mathrm{discussed} \mathrm{in}$ $\S 5.2$.

\section{RAPID NONTHERMAL PARTICLE ACCELERATION ON LATE-TYPE STARS}

\subsection{Small Sizes, High Brightness Temperatures, and Coherent Radiation}

Powerful (up to $200 \mathrm{mJy}$ ), impulsive (a few minutes), highly circularly polarized $(100 \%)$ radio bursts are emitted from dwarf $M$ flare stars, often with rapid rise times that provide stringent limits to the size of the emitting source and indicate coherent radiation processes. Radio flares from the dwarf $M$ star AD Leo near $20 \mathrm{~cm}$ wavelength consist, for example, of rapid (less than 100 milliseconds), $100 \%$ circularly polarized spikes that were first observed by Lang et al. (1983-see Fig. 5) using the Arecibo Observatory, and confirmed by Lang \& Willson (1986b) and Bastian et al. (1990) using the same radio telescope, and by Güdel et al. (1989) and Benz et al. (1990)

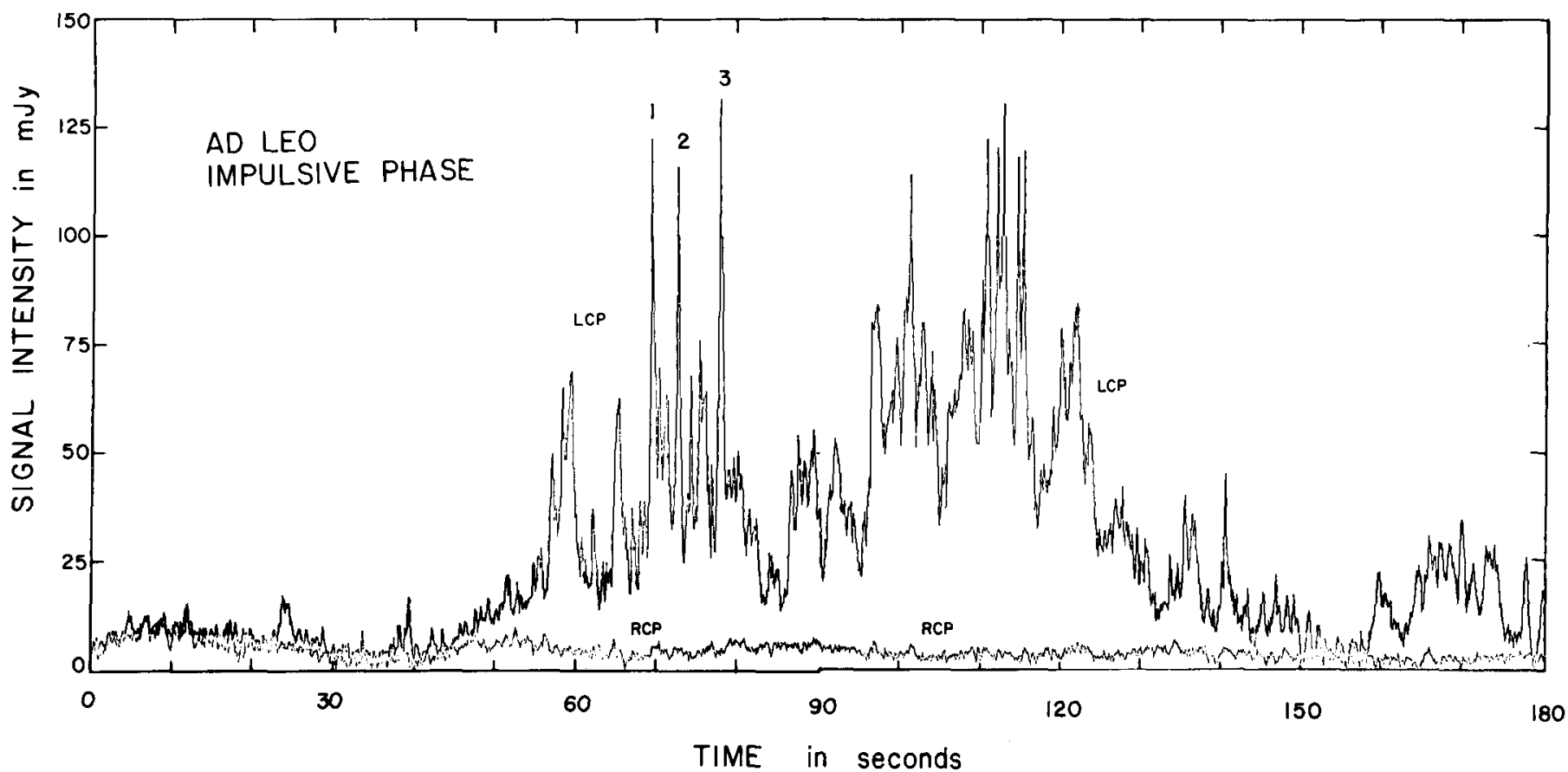

FIG. 5.- Rapid, highly polarized spikes observed at $20 \mathrm{~cm}$ wavelength during a radio burst, or flare, from the dwarf $\mathrm{M}$ flare star AD Leonis. The spikes are $100 \%$ left-hand circularly polarized ( LCP). Those labeled 1,2, and 3 had rise times of less than 200 milliseconds; the light-travel time argument indicates that the emitter is smaller than the star in size. Provided that the source is symmetric, it has an area less than 0.03 of the star's surface area, and a brightness temperature in excess of ten thousand billion degrees. (Adapted from Lang et al. 1983, also see Lang \& Willson 1986b.) 
whose simultaneous observations with the radio telescopes in Effelsberg, Jodrell Bank, and Arecibo eliminated any remaining doubts about its stellar origin.

Highly circularly polarized radio bursts of short duration have also been observed from active binary stars of late spectral type, including RS CVn stars (Brown \& Crane 1979; Mutel et al. 1987) and Algol (Lestrade et al. 1988), suggesting radio emission of coherent origin. VLBI imaging indicates that these flaring regions may be considerably smaller than either visible component of these binary systems.

Sizes and brightness temperatures can be inferred from the rise times of spikes detected during radio bursts on dwarf $M$ stars. The spikes labeled 1,2, and 3 in Figure 5 have rise times of less than 200 milliseconds, and upper limits of 20 milliseconds have been observed. A light-travel time of 20 milliseconds indicates a size of less than 6,000 kilometers, which is less than $1 \%$ of the stellar diameter. If the burst emitter is symmetric, it has an area less than 0.0003 of the stellar surface area, and a brightness temperature greater than $10^{15} \mathrm{~K}$.

Such high brightness temperatures require a coherent radiation mechanism, and the high circular polarization indicates an intimate connection with the stellar magnetic fields. One possible explanation is the electron-cyclotron maser that has been adapted to explain millisecond spikes on the Sun (see $\S 2.3$ and Melrose \& Dulk 1982). Coherent plasma radiation is another possibility. Both mechanisms require a strong coronal magnetic field to produce the high circular polarization-either at the site of radiation or during subsequent propagation of initially unpolarized radiation.

The coherent radiation processes provide constraints on the physical conditions in flare stars. An upper limit to the electron density, $N_{e}$, in the source is given by the requirement that the observing frequency must be greater than the plasma frequency for the radiation to propagate out and reach the observer; at $20 \mathrm{~cm}$ wavelength this requires $N_{e} \leq 2.5 \times 10^{10}$ $\mathrm{cm}^{-3}$. If an electron-cyclotron maser emits at the second harmonic of the gyrofrequency, then a coronal magnetic field strength of $H=250 \mathrm{G}$ is required to explain the $20 \mathrm{~cm}$ radiation; lower magnetic field strengths are needed if coherent plasma radiation dominates.

\subsection{Quasi-Periodic Fluctuations and Sudden Reductions}

Quasi-periodic fluctuations during flares on some dwarf $M$ stars suggest pulsations or oscillations that modulate the radio emission. Broad-band ( $100 \mathrm{MHz}$ or more), quasi-periodic (about 1 second period), 100\% circularly polarized radio fluctuations have been reported for the dwarf $\mathrm{M}$ star AD Leo by Lang \& Willson (1986c), Gudel et al. (1990) and Bastian et al. (1990-see Fig. 6). Highly circularly-polarized radio flux variations have also been observed for the dwarf $M$ binary system UV Ceti, but with a longer period of about 56 seconds (Gary,

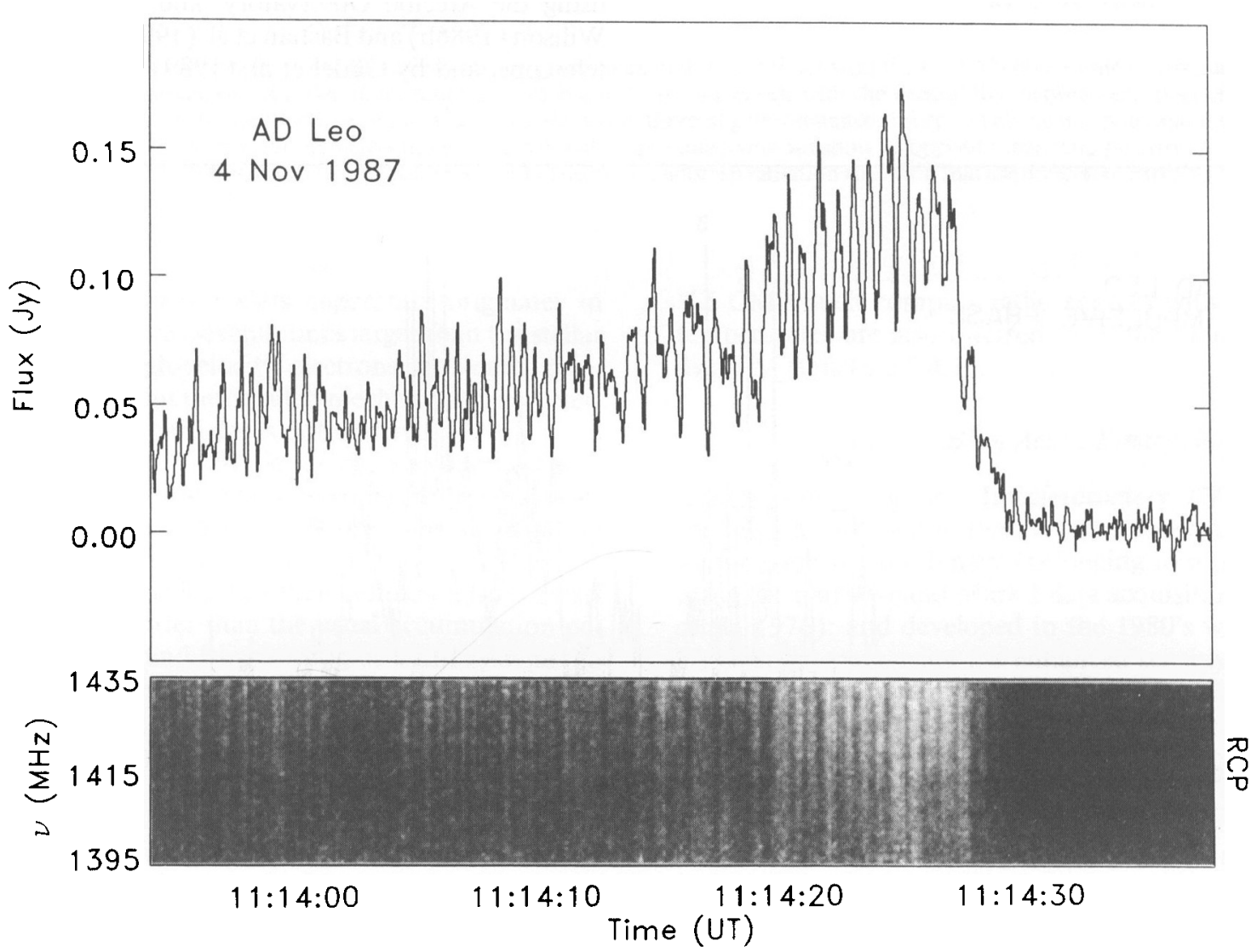

Fig. 6.-Broad-band pulsations from the dwarf $\mathrm{M}$ flare star $\mathrm{AD}$ Leonis at $20 \mathrm{~cm}$ wavelength. The radiation is $100 \%$ right-hand circularly polarized (RCP); the RCP dynamic spectra are shown at the bottom. The pulsations show an amplitude modulation of about $50 \%$ and a period of about 0.7 seconds near the burst maximum; the radio emission suddenly ceases after this maximum. (Adapted from Bastian et al. 1990.) 
Linsky \& Dulk 1982). Similar fluctuations have been observed during solar radio bursts, and interpreted in terms of a modulation of particle acceleration and/or injection, or oscillations in a flux tube (see $\$ 2.3$ ). Sudden reductions in flare star radio emission ( see Fig. 7) also have a solar analogy (see $\$ 2.3$ ).

\subsection{Dynamic Radio Spectra of Late-Type Stars}

Observations of the radio radiation intensity as a function of both time and frequency (dynamic spectra) provide unique information about particle acceleration on dwarf $M$ flare stars. Such dynamic spectra have been obtained for AD Leo, YZ CMi and UV Ceti, showing both narrow-band and broad-band features and both positive and negative frequency drifts (Bastian \& Bookbinder 1987; Jackson, Kundu, \& White 1987; Bastian et al. 1990). Positive frequency drifts were observed for UV Ceti, for example, suggesting a disturbance that propagates downward in the stellar corona and progressively excites plasma radiation at higher frequencies (larger electron densities); Bookbinder et al. (1992) have obtained dynamic ultraviolet spectra of AD Leo using the Hubble Space Telescope, also indicating high-speed infall of gas during a flare. The example shown in Figure 7 has a negative drift of $250 \mathrm{MHz}$ per second from high to low frequencies, suggesting electron beams that propagate outward in the stellar corona at nearly the velocity of light; such drifts are commonly observed in the Sun during type III bursts ( see $\$ 2.4$ ).

\section{LONG-LIVED NONTHERMAL PARTICLE ACCELERATION ON LATE-TYPE STARS}

\subsection{Long-Lasting Radio Emission from Dwarf M Flare Stars}

Radio radiation from some dwarf $M$ flare stars is nearly always present, only slowly varying with time, and it is sometimes unpolarized. It has been termed quiescent radio radiation to distinguish it from the brief radio flares that are often highly circularly polarized. However, we do not know if the dwarf $M$ stars have a real quiescent state, or if the persistent radio emission is due to low-amplitude flare events that occur on a more or less continuous basis. The more important point is that the steady, long-lived radio radiation requires the continued acceleration of nonthermal electrons over periods of hours, and the explanation for this sustained particle acceleration remains unknown.

Although thermal gyroresonance radiation might account for the long-lived radio emission at short wavelengths (Güdel \& Benz 1989), it cannot explain the long-wavelength radio radiation whose observed flux densities would require implausibly intense magnetic fields in sources that are tens to hundreds of times larger than the visible star (Lang 1990b). The persistent radio radiation of dwarf $M$ stars has been more commonly attributed to the nonthermal gyrosynchrotron radiation of mildly relativistic electrons (Kundu \& Shevgaonkar 1985; Pallavicini, Willson, \& Lang 1985).

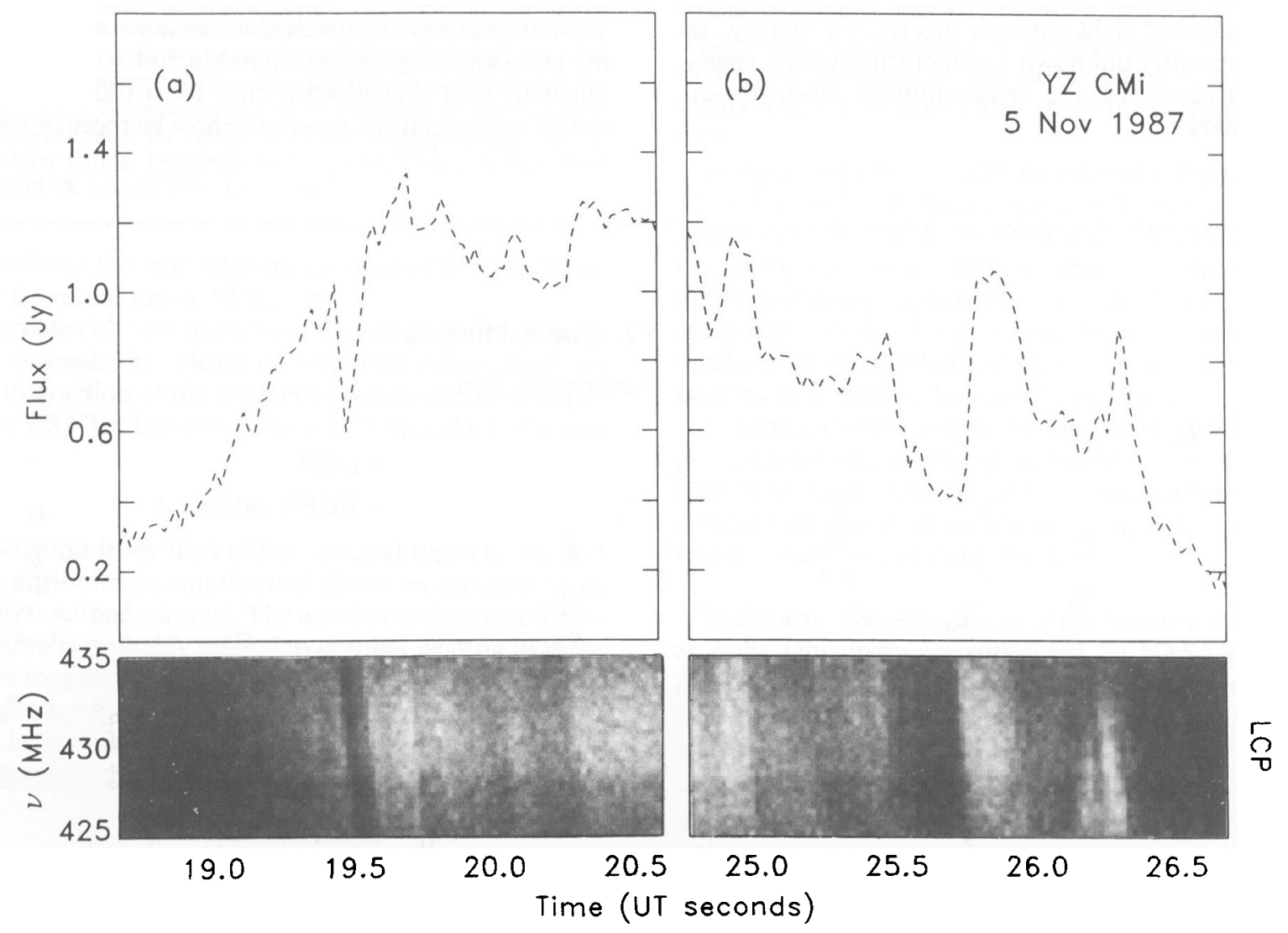

FIG. 7. - "Sudden reduction" feature ( $t o p$ ) in the $100 \%$ left-hand circularly polarized (LCP) radiation of the dwarf M flare star YZ Canis Minoris at 430 $\mathrm{MHz}$ exhibits a drift in frequency of about $250 \mathrm{MHz}$ per second from high to low frequencies (bottom). (Adapted from Bastian et al. 1990.) 
Detailed gyrosynchrotron models of the long-lasting radio emission have been provided by White, Kundu, \& Jackson $(1989 \mathrm{a}, 1989 \mathrm{~b})$, assuming that the emitting electrons have a power-law energy distribution with a spectral index around 3. They argue that the hot plasma on dwarf $M$ flare stars is confined within coronal loops anchored in active regions, much as it is in the Sun, and that the nonthermal gyrosynchrotron emission originates in several small active regions rather than a global dipolar magnetic structure. If this scenario is correct, then some unknown process must be nearly continuously accelerating electrons to energies of tens to hundreds of $\mathrm{keV}$ and injecting them into the coronal atmosphere above stellar active regions.

A different interpretation is required for relatively intense, narrow-band radio radiation lasting for several hours; nonthermal gyrosynchrotron emission is not expected to have a narrow bandwidth. Such long-lived, narrow-band radiation has been observed for several dwarf $M$ flare stars (Lang \& Willson 1986c; White, Kundu, \& Jackson 1986; Kundu et al. 1987; Lang \& Willson 1988). It has been observed to be up to $100 \%$ circularly polarized, and to have a fractional bandwidth of 0.02 (Fig. 8; Lang \& Willson 1988). The narrow-band radio emission may be due to coherent mechanisms like electron-cyclotron masers or coherent plasma radiation.

Different radiation mechanisms might dominate at different wavelengths. Coherent emission processes may dominate at long wavelengths above $10 \mathrm{~cm}$, while incoherent gyrosynchrotron emission might be the dominant radiation mechanism at shorter wavelengths where coherent emission is limited due to a maximum magnetic field strength or electron density. In either case, a currently unknown mechanism must be nearly continuously accelerating the radio-emitting electrons on dwarf $M$ flare stars.

\subsection{Models of Long-Lasting Radio Radiation from $R S C V n$ Stars}

A variety of models have been developed to explain the longlived (hours) radio emission from RS CVn stars including coherent electron-cyclotron maser radiation from core sources smaller than the visible star (Lestrade et al. 1988), synchrotron radiation from relativistic electrons (Mutel et al. 1985), and the gyrosynchrotron radiation of mildly relativistic electrons in a halo that is comparable in size to the separation of the binary stars (Owen, Jones, \& Gibson 1976; Klein \& ChiuderiDrago 1987).

Long-lasting radio radiation with high circular polarization has been observed from RS CVn stars, and Willson \& Lang (1987) have shown that variable radio emission lasting for hours can have a fairly narrow bandwidth (detectable at 4835 $\mathrm{MHz}$ and not detected at $1415 \mathrm{MHz}$ ). So, the sort of long-lasting, coherent radio radiation detected from dwarf $M$ stars might occasionally arise on RS CVn stars as well.

But the more developed models for the long-lasting radio emission from RS CVn stars involve gyrosynchrotron emission from nonthermal electrons characterized by a power-law distribution of energies. They are based upon observations of moderate, persistent circular polarization, extents comparable to the size of the binary system, brightness temperatures of $10^{8}$ to $10^{9} \mathrm{~K}$, and a nearly flat spectrum at centimeter wavelengths. Morris, Mutel, \& Shu (1990) have explained these observations in terms of gyrosynchrotron radiation in a toroidal region, or "dead zone," of plasma trapped within a stable, largescale magnetosphere attached to the more active star (Fig. 9); the radio emitting regions extend to 5 or 10 stellar radii where magnetic field strengths are from 10 to $100 \mathrm{G}$. In this model, nonthermal electrons must somehow be more or less continu-

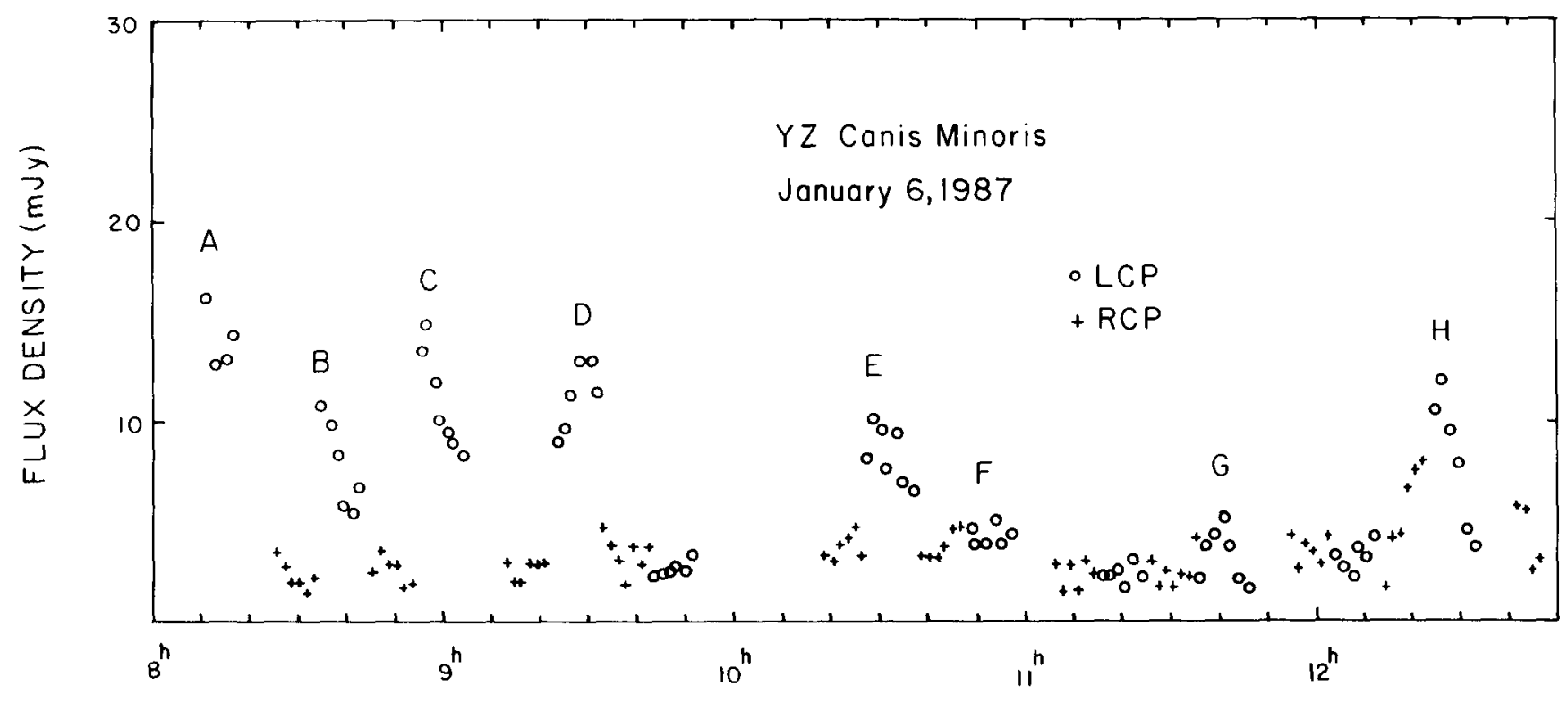

UNIVERSAL TIME

FiG. 8.-Slowly varying, long-lasting (hours), $100 \%$ left-hand circularly polarized (LCP) radiation from the dwarf M flare star YZ Canis Minoris at 20 $\mathrm{cm}$ wavelength. Frequency spectra of the 10 minute intervals marked by the letters $\mathrm{A}, \mathrm{B}, \ldots \mathrm{H}$ exhibited narrow-band structure with a fractional bandwidth of 0.02 . (Adapted from Lang \& Willson 1988.) 

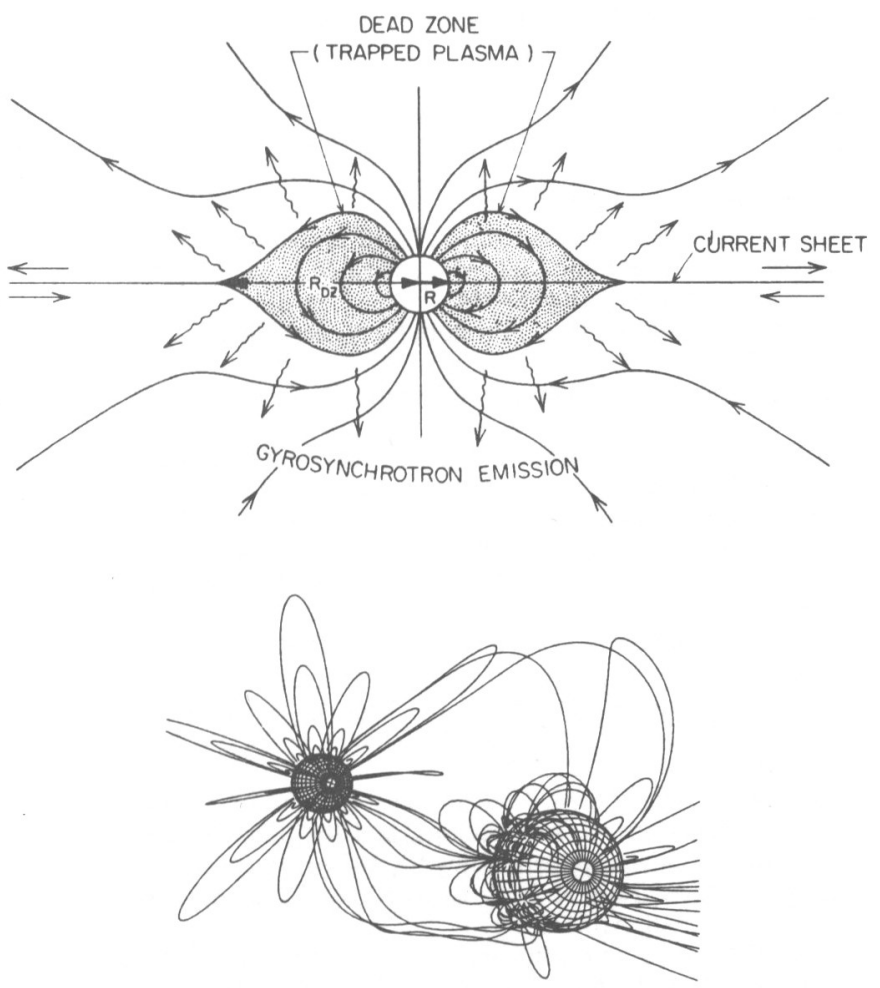

FIG. 9.-Quiescent radio radiation from binary $\mathrm{RS} C V \mathrm{n}$ stars may be due to the gyrosynchrotron radiation of electrons accelerated to relativistic energies, and trapped within a dipolar magnetic "dead zone" that is several times the radius of the active stellar component ( $t o p)$. An extended corona of relativistic electrons might also be built up through the interaction of the magnetospheres of both components of the RS CVn binaries (bottom). In either case, long-lived (hours to days), nonthermal particle acceleration is required, perhaps through the release of stored magnetic energy during repeated low-level flaring. [Adapted from Morris, Mutel, \& Shu 1990 (top), and Uchida \& Sakurai 1983 (bottom).]

ously injected into the vast magnetic coronae of RS CVn stars, perhaps by persistent low-level flaring.

In an alternate view, extended coronae and particle acceleration occur through the release of magnetic energy built up during the interaction of the magnetospheres on both components of the RS CVn binaries (also Fig. 9; Uchida \& Sakurai 1983).

\section{SUMMARY}

Radio radiation from stars of late spectral type $(G, K$, and M) can be attributed to nonthermal electrons accelerated in magnetically confined coronae. The nonthermal particle acceleration process is probably related to coronal heating in these stars, and to magnetic activity resulting from internal rotation and convection.

The Sun is the only star close enough to resolve the detailed magnetic structure during radio flares. VLA data indicate that radio flares often occur at the apex of coronal loops where particle acceleration often occurs, and that different flare phases can occur in nearby, but spatially separate, coronal loops.

The observational and theoretical framework for interpreting particle acceleration on late-type stars is provided by the Sun, including long-lasting thermal bremsstrahlung and thermal gyroresonance emission, nonthermal solar radio bursts, coherent microwave spike bursts, quasi-periodic variations, sudden reductions, positive and negative frequency drifts, and recent evidence for long-lasting nonthermal radiation.

VLBI observations provide evidence for nonthermal particle acceleration on single pre-main-sequence $\mathrm{T}$ Tauri stars and on binary RS CVn stars. High-velocity electrons can be nearly continuously accelerated on these stars within large-scale magnetospheres that are several times larger than the radius of a visible star. In contrast, VLBI of dwarf $\mathrm{M}$ stars suggest radio sources smaller in size than the visible star.

Rapid rise times of highly circularly polarized radio bursts from some dwarf $M$ stars require small sizes with an area less than 0.0003 of the stellar surface area, and brightness temperatures exceeding $10^{15} \mathrm{~K}$. This radio emission might be explained by coherent processes such as an electron-cyclotron maser or coherent plasma radiation; these mechanisms provide constraints on the coronal magnetic field strength $H=$ $250 \mathrm{G}$ and the electron density $N_{e} \leq 2.5 \times 10^{10} \mathrm{~cm}^{-3}$.

Radio radiation from dwarf $\mathrm{M}$ flare stars also exhibits quasiperiodic fluctuations, sudden reductions and positive and negative frequency drifts. The negative frequency drifts to lower frequencies suggest particle acceleration by electron beams that travel outward in the stellar corona; positive drifts to higher frequencies imply high-speed infall of particles during a flare.

Long-lasting (hours) radio emission from dwarf $\mathrm{M}$ stars can be explained by the incoherent nonthermal gyrosynchrotron radiation of several small active regions, and/or by coherent nonthermal processes. Both mechanisms might be operating and, respectively, dominating the radiation at short and long radio wavelengths. They require the more-or-less continuous acceleration of nonthermal electrons over periods of hours, perhaps by persistent low-amplitude flares.

Similar, currently unknown, long-lived particle acceleration processes are required to explain radio radiation from $\mathrm{RSCVn}$ stars. It has been attributed to the gyrosynchrotron emission of nonthermal electrons in stable, large-scale magnetospheres that are many stellar radii in extent.

Radio astronomical studies of the Sun and other active stars at Tufts University are supported by NASA grant NAGW2383. The VLA-RATAN 600 solar observations are supported by NSF grant ATM-9024506 and NATO collaborative research grant CRG 921394.

\section{REFERENCES}

Akhmedov, Sh. B., et al. 1986, ApJ, 301, 460

Alef, W., Benz, A. O., Güdel, M., \& de Vicente, P. 1993, in preparation Alissandrakis, C. E., \& Kundu, M. R. 1982, ApJ, 253, L49

André, P., Deeney, B. D., Phillips, R. B., \& Lestrade, J.-F. 1992, ApJ, 401, 667
André, P., Montmerle, T., \& Feigelson, E. D. 1987, AJ, 93, 1182

André, P., Phillips, R. B., Lestrade, J.-F., \& Klein, K.-L. 1991, ApJ, 376, 630

Bastian, T. S. 1990, Sol. Phys, 130, 265

Bastian, T. S., \& Bookbinder, J. A. 1987, Nature 326, 678 
Bastian, T. S., Bookbinder, J., Dulk, G. A., \& Davis, M. 1990, ApJ 353 265

Benz, A. O., \& Alef, W. 1991, A\&A, 252, L19

Benz, A. O., Gudel, M., Bastian, T. S., Furst, E., Simnett, G. M., \& Pointon, L. 1990, in IAU Symp. 137, Flare Stars in Star Clusters, Associations, and the Solar Vicinity, ed. L. V. Mirzoyan et al. (Dordrecht: Kluwer), 139

Benz, A. O., \& Kuijpers, J. 1976, Sol. Phys., 46, 475

Bogod, V. M., Gelfreikh, G. B., Willson, R. F., Lang, K. R., Opeikina, L. V., Shatilov, V., \& Tsvetkov, S. V. 1992, Sol. Phys., 141, 303

Bookbinder, J. A., et al. 1992, S\&T, 83, 625

Brown, R. L., \& Crane, P. C. 1979, AJ 83, 1504

Clark, T. A., et al. 1976 ApJ, 206, L107

Drake, S. A., Simon, T., \& Linsky, J. L. 1989, ApJS, 71, 905 1992, ApJS, 82, 311

Fiegelson, E. D., Giampapa, M. S., \& Vrba, F. J. 1991, in The Sun in Time, ed. C. P. Sonett, M. S. Giampapa, \& M. S. Matthews (Tucson: Univ. Arizona Press), 658

Gary, D. E., Linsky, J. L., \& Dulk, G. A. 1982, ApJ, 263, L69

Güdel, M. 1992, A\&A, 264, L31

Güdel, M.. \& Benz, A. O. 1989, A\&A, 211, L5 1993, ApJ, 405, L63

Güdel, M., Benz, A. O., Bastian, T. S., Furst, E., Simnett, G. M., \& Davis, R. J. 1989, A\&A, 220, L5

Güdel, M., et al. 1993, in preparation

Haisch, B., Strong, K. T., \& Rodono, M. 1991, ARA\&A, 29, 275

Hjellming, R. M. 1988, in Galactic and Extragalactic Radio Astronomy, ed. G. L. Verschuur \& K. I. Kellermann (New York: Springer), 381

Jackson, P. D., Kundu, M. R., \& White, S. M. 1987, ApJ, 316, L85

1989, A\&A, 210,284

Klein, K.-L., \& Chiuderi-Drago, F. 1987, A\&A, 175, 179

Kuijpers, J., 1989, Sol. Phys., 121, 163

Kundu, M. R., Jackson, P. D., White, S. M., \& Melozzi, M. 1987, ApJ, 312,822

Kundu, M. R., \& Lang, K. R. 1985, Science, 228, 9

Kundu, M. R., Schmahl, E. J., Velusamy, T., \& Vlahos, L. 1982, A\&A, 108,188

Kundu, M. R., \& Shevgaonkar, R. K. 1985, ApJ, 297, 644 1988, ApJ, 334, 1001

Lang, K. R. 1986, Adv. Space Res., 6, No. 8, 109

. 1990a, in IAU Symp. 142, Basic Plasma Processes on the Sun, ed.

E. R. Priest \& V. Krishan (Dordrecht: Kluwer), 501

. 1990b, in IAU Symp. 137, Flare Stars in Star Clusters, Associations and the Solar Vicinity, ed. L. V. Mirzoyan et al. (Dordrecht: Kluwer), 125 1991, Astrophysical Data: Planets and Stars (New York: Springer)

Lang, K. R., Bookbinder, J., Golub, L., \& Davis, M. M. 1983, ApJ, 272, L15

Lang, K. R., \& Willson, R. F. 1982, ApJ, 255, L111

. 1986a, Adv. Space Res., 6, No. 6, 97
Lang, K. R., \& Willson, R. F. 1986b, ApJ, 305, 363

1986c, ApJ, 302, L17

1988, ApJ, 326, 300

Lang, K. R., et al. 1993, ApJ, in press

Lang, K. R., Willson, R. F., Smith, K. L., \& Strong, K. T. 1987a, ApJ, 322, 1035

. 1987b, ApJ, 322, 1044

Lestrade, J.-F. 1988, in IAU Symp. 129, in The Impact of VLBI on Astrophysics and Geophysics, ed. M. J. Reid \& J. M. Moran (Dordrecht: Reidel), 265

Lestrade, J.-F., Mutel, R. L., Preston, R. A., \& Phillips, R. B. 1988, ApJ, 328,232

Lim, J., Nelson, G. J., Castro, C., Kilkenny, D., \& van Wyk, F. 1992, ApJ, $388, \mathrm{~L} 27$

McLean, D. J., \& Labrum, N. R. 1985, Solar Radiophysics (New York: Cambridge Univ. Press)

Melrose, D. B., \& Dulk, G. A. 1982, ApJ, 259, 844

Morris, D. H., Mutel, R. L., \& Shu, B. 1990, ApJ, 362, 299

Mutel, R. L., Lestrade, J.-F., Preston, R. A., \& Phillips, R. B. 1985, ApJ, 289,262

Mutel, R. L., Morris, D. H., Doiron, D. J., \& Lestrade, J.-F. 1987, AJ, 93, 1220

Owen, F. N., Jones, T., \& Gibson, D. 1976, ApJ, 210, L27

Pallavicini, R., Golub, L., Rosner, R., Vaiana, G. S., Ayres, T., \& Linsky, J. L. 1981, ApJ, 248, 279

Pallavicini, R., Stella, L., \& Tagliaferri, G. 1990, in IAU Symp. 137, Flare Stars in Star Clusters, Associations, and the Solar Vicinity, ed. L. V. Mirzoyan et al. (Dordrecht: Kluwer), 147

Pallavicini, R., Willson, R. F., \& Lang, K. R. 1985, A\&A, 149, 95

Parker, E. N. 1988, ApJ, 330, 474

Phillips, R. B. 1992, in Cool Stars, Stellar Systems, and the Sun, ed. M. S. Giampapa \& J. A. Bookbinder (ASP Conf. Ser., 26), 309

Phillips, R. B., Lonsdale, C. J., \& Feigelson, E. D. 1991, ApJ, 382, 261 . 1993, ApJ, 403, L43

Roberts, B., Edwin, P. M., \& Benz, A. O. 1984, ApJ, 279, 858

Slee, O. B., \& Stewart, R. T. 1989, MNRAS, 236, 129

Stine, P. C., Feigelson, E. D., Andre, P., \& Montmerle, T. 1988, AJ, 96, 1394

Sturrock, P. A., Dixon, W. W., Klimchuk, J. A., \& Antiochos, S. K. 1990 ApJ, 365, L31

Uchida, V., \& Sakurai, T. 1983, in IAU Colloq. 71, Activity in Red Dwarf Stars, ed. M. Rodono \& P. Byrne (Dordrecht: Reidel), 629

White, S. M., Jackson, P. D., \& Kundu, M. R. 1989a, ApJS, 71, 895 $1989 \mathrm{~b}$, A\&A, 199, 255

White, S. M., Kundu, M. R., \& Jackson, P. D. 1986, ApJ, 311,814

White, S. M., Pallavicini, R., \& Kundu, M. R. 1992a, A\&A, 259, 149 1992b, A\&A, 259, 149

Willson, R. F., \& Lang, K. R. 1987, ApJ, 312, 278

Willson, R. F., Lang, K. R., \& Liggett, M. 1990, ApJ, 350, 856 